\title{
Power Flow Automatic Adjustment Based on Reactive Power Distribution Factor
}

\author{
Xiang Xiao-rong ${ }^{1, a}$, Liu Di-chen ${ }^{2, b}$, Wang Bo ${ }^{3, c}$, Xiang Nong ${ }^{4, d}$ \\ ${ }^{1,2,3}$ School of Electrical Engineering, Wuhan University, Wuhan, 430072, China \\ a654256164@qq.com, bdcliu@whu.edu.cn
}

Keywords: reactive power; active power; reactive power distribution factor; automatic adjustment algorithm

\begin{abstract}
On account of the regional balance requirements of reactive power, this paper proposes automatic adjustment method based on reactive power distribution factor for the trend. In this paper, the equivalent impedance of the $\pi$-type equivalent circuit is as the electrical distance to strike the reactive power allocation factor. In the Reactive iterative process, node reactive power mismatch is compensated according to reactive power balance rules and distribution factor. Furthermore, based on the PQ decomposition method, automatic adjustment algorithms of the active and reactive power are added to the iterative process, the system active and reactive power vacancies are assigned to generator nodes in the system which still have the capacity of accommodation in accordance with their adjustment strategy to realize automatic adjustment; in the later period of iterative process, power flow calculation is translated to the regular trend to promote the rapid convergence of the whole algorithm. The results show that the system load changes, under the premise of meeting the node voltage and reactive power constraints the proposed method can rationally allocate the power flow and achieve the automatic adjustment.
\end{abstract}

\section{Introduction}

After the power flow system's operation mode is adjusted (such as modified power generation, load, network topology, etc), the system's power flow may not keep balance. Our country electric power network has high pressure, high capacity, long-distance transmission and other characteristics. In the bulk power systems, the most (70-80\%) areas can keep a good trend results, and the situation that power flow can't converge just happens in the local area and mainly caused by the unbalance of reactive power.

The power flow calculation is the most basic work of power system analysis, for decades, a large number of documents have proposed many flow algorithms. In general, they include regular flow algorithm $^{[1-4]}$ and optimal power flow algorithm based on different targets ${ }^{[5-10]}$. The optimal power flow analysis ${ }^{[1-14]}$ although can realize the on-site reactive power's balance, but calculation is so complex that the efficiency is decreased. At the same time, the trend of the conventional calculation's efficiency although can be sure, but the reactive power's balance depends on the selecting of PV nodes and balance nodes, it is too artificial to guarantee the on-site reactive power balance. Literature [15] expanded the regular flow algorithm by defining the new node types which made the reactive power have the function of on-site local balance, which largely inherited the ability that the optimal power flow can optimize the distribution of the reactive power, also has won with regular flow algorithm quite calculation efficiency. But literature [15] defined new node which made the algorithm complex, increased the complexity of the calculation, reduced the computational efficiency.

In this paper, on the basis of document [15], the power flow's automatic adjusting method based on reactive power distribution factor is put forward. According to the principle of on-site reactive power balance requirements, for the nodes, they base the electric distance on the equivalent impedance which get from the $\pi$ model equivalent circuit model, and on this basis reactive power distribution factor is obtained. Then according to the reactive power distribution factor, the systems distribute the node's unbalanced reactive power to the reactive power generation node, and adjust 
their own reactive power, and gradually reduce the node's reactive power which is out of balance, so that all of the nodes' reactive power can balance on the basis of on-site reasonable distribution. Finally the IEEE30 nodes system is for example, and verified the proposed method which is called as the power flow's automatic adjusting based on reactive power distribution factor, and this paper also compared results of this algorithm with regular flow algorithm's. The results show that the algorithm can effectively achieve automatic adjustment of the trend and is more rational and superiority.

\section{Define bus types}

The bus types' definition is the same as conditional trend's, and divide into three kinds of nodes:

(1) The PQ bus: the active power $\mathrm{P}$ and reactive power $Q$ is given, bus voltage and phase $(V, \delta)$ stays for unknown. Usually electric power substation belongs to this type bus. Since there is no power generation equipment, so the generated power is zero. In some cases, the power which is sent out by some of the power stations in the system is a fixed value in a certain time, the plant is also as PQ bus, therefore, in power system, most nodes belong to this type.

(2) The PV bus: the active power $P$ and voltage amplitude $V$ is given, the reactive power $Q$ and voltage phase $\delta$ is unknown. This kind of bus must have enough adjustable reactive capacity to sustain a given voltage amplitude, and also is called as "the voltage control bus". Generally, power plants which have certain reactive reserve and power substations which are installed a adjustable reactive power equipment are chose as PV bus. In the power system, this kind of node is a little number.

(3) The swing bus's voltage amplitude and phase is given, and the injection active power and reactive power is unknown.

\section{The mathematical model of trend automatically adjusting}

\section{Reactive power distribution factor}

Reactive power distribution factor (hereinafter referred to as the distribution factor) is determined by the electric distance.

The electric distance has a variety of definition, literature $[16,17]$ used the sensitivity of a node voltage's change to another node reactive power's to definite the electric distance between two nodes, the literature [18] adopted the transfer admittance between nodes as the electrical distance, the former is influenced bigger by running state of electric power system, the transfer admittance matrix of the latter is sparse, and can't fully reflect the electrical connection between the nodes.

In this paper, the equivalent impedance $Z_{i j}=r_{i j}+j x_{i j}$ of the node $i$ and $j$ between the $\pi$-type equivalent circuit defines the electrical distance between nodes $i$ and $j$, and on this basis to strike the reactive power distribution factor.

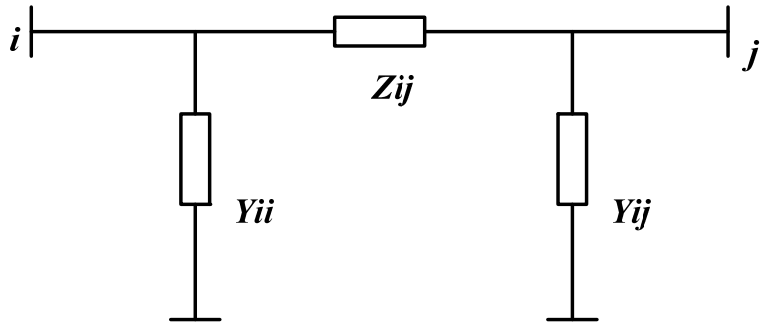

Fig. 1. The $\pi$-type equivalent circuit between nodes $i$ and $j$

For the transmission grid, owing to $r_{i j} \ll x_{i j}$, therefore the delivery reactive power of the node $i$ to node $j$ has the following approximate relationship:

$$
Q_{i j}=\frac{U_{i}\left(U_{i}-U_{j}\right)}{x_{i j}}
$$

Equation (1) shows that the equivalent reactance between nodes $i$ and $j$ is inversely proportional 
to the transmission reactive power. Could be envisaged, reactive power support which the rest of reactive power generation node of node $i$ surrounding that can provide exist a simple inverse relationship with the equivalent reactance. Namely the allocation factors are inversely proportional to line equivalent reactance.

Based on the equivalent impedance, select several reactive power generation nodes that contact most closely (equivalent reactance is less) to node $i$ compose of a collection $\Omega_{i}$ which is as a reactive power balance area of node $i$. In the region, the $k$-th reactive power distribution factor for node $i$ as follows:

$$
\lambda_{k i}=\frac{1 / \lambda_{k i}}{\sum_{j \in \Omega_{i}} 1 / \lambda_{k i}},\left(k \in \Omega_{i}\right)
$$

\section{Rules of the balance of reactive power mismatch}

Reactive power generation nodes to balance the reactive power must follow certain rules. Node $\mathrm{i}$ balanced regional controlled conditions as follows: In accordance with the distribution factor for allocation of reactive power mismatch, the voltage and reactive of power generation node power can't be out of limit. The mathematical expression of the balance rules of the mismatch is:

$$
\left(Q_{G i}+\Delta Q_{G i}\right) \in\left\{\left[Q_{G i \min }, Q_{G i \max }\right] \cap\left[U_{G i \min }, U_{G i \max }\right]\right\}
$$

Where, $Q_{G i}$ is initial reactive power of node $i, \Delta Q_{G i}$ is mismatches in accordance with the reactive power allocation factor assigned to node $i,\left[Q_{G i \min }, Q_{G i \max }\right]$ is node $i$ reactive restrictions, $\left[U_{G i \min }, U_{G i \max }\right]$ is node $\mathrm{i}$ voltage restrictions.

\section{Power flow equations}

Establish the load flow equations:

$$
\begin{aligned}
& Q_{\mathrm{Gi}}^{(t)}=\Delta Q_{\mathrm{Gi}}^{(t-1)}+\sum_{\mathrm{k} \in n} \lambda_{k i} \Delta Q_{k}^{(t-1)} \\
& \Delta Q_{\mathrm{i}}^{(t)}=Q_{\mathrm{Gi}}^{(t)}-Q_{\mathrm{Li}}^{(t)}-V_{i} \sum_{j \in i} V_{j}\left(G_{i j} \sin \theta_{i j}-B_{i j} \cos \theta_{i j}\right) \\
& \Delta P_{\mathrm{i}}^{(t)}=P_{\mathrm{Gi}}^{(t)}-P_{\mathrm{Li}}^{(t)}-V_{i} \sum_{j \in i} V_{j}\left(G_{i j} \cos \theta_{i j}+B_{i j} \sin \theta_{i j}\right)
\end{aligned}
$$

Where, $\Delta Q_{\mathrm{i}}^{(t)}$ and $\Delta P_{\mathrm{i}}^{(t)}$ are the reactive and active power mismatch of node $i$;

$P_{\mathrm{Gi}}^{(t)}$ and $Q_{\mathrm{Gi}}^{(t)}$ are active and reactive power output of node i (for non-generator nodes, and its value is equal to 0 );

$P_{\mathrm{Li}}^{(t)}$ and $Q_{\mathrm{Li}}^{(t)}$ are active and reactive power load of node i (for non-load nodes, and its value is equal to 0 );

$V_{i}$ and $V_{j}$ are node $i$ and $j$ voltage amplitude, the two node voltage phase difference is $\theta_{i j}$;

$G_{i j}$ and $B_{i j}$ are the real and imaginary parts of the $i$-th row $j$-th column elements of node admittance matrix.

\section{Correction equation}

Correction equation using the $P-Q$ decomposition method as follows:

$$
\left[\begin{array}{c}
\Delta P \\
\Delta Q
\end{array}\right]=-\left[\begin{array}{cc}
H & N \\
K & L
\end{array}\right]\left[\begin{array}{c}
\Delta \delta \\
V_{D_{2}}^{-1} \Delta V
\end{array}\right]
$$

In the $\mathrm{AC}$ high voltage power grid, transmission line reactance is much greater than resistance and bus active power in the system changes with the voltage phase, the reactive power is mainly affected by the change of bus voltage amplitude. In the coefficient matrix of correction equation, the value of partial derivatives

$\frac{\partial \Delta P}{\partial \Delta V}$ and $\frac{\partial \Delta Q}{\partial \Delta \delta}$ relative to the partial derivative $\frac{\partial \Delta P}{\partial \Delta \delta}$ and $\frac{\partial \Delta Q}{\partial \Delta V}$ is very small, so equation's 
sub-block is negligible, can be considered their elements are equal to zero. Simplify $H$ and $L$ as a constant matrix, decomposition can be obtained:

$$
\left\{\begin{array}{c}
\Delta P=-\mathrm{V}_{D 1} \mathrm{~B}^{\prime} \mathrm{V}_{D 1} \Delta \delta \\
\Delta Q=-\mathrm{V}_{D 2} \mathrm{~B}^{\prime \prime} \Delta V
\end{array}\right.
$$

Where B' matrix of order $n-1$, excluding the balance node's corresponding rows and columns; B" matrix of order $m$, no balancing node and the corresponding rows and columns of the $P V$ nodes.

\section{Handover with the conventional trend}

Automatic adjustment process based on reactive power distribution factor for the trend to a certain degree, the system's accelerating power had been fully allocated between the generator nodes, and got the final stable operating frequency, the reactive power of the various regions are basically situ balance, the remaining small fraction of reactive power mismatch has little effect on computing the final result of the trend. At this time, in order to speed up the convergence process, a one-time processing can make the iterative process transit to a conventional power flow calculation. Specific conversion is as follows :

First, the phase angle reference node remains the reference role, and retains its current voltage amplitude and acts in the conventional power flow as the balance node meanwhile.

Then the generator nodes outside balance node are handled as follows: active power current value is retained and fixed, for the generator nodes that reactive power is out of limit, its reactive power will be placed in the upper limit and adopt the methodology used in the joint adjustment to process the appropriate handling, then the node will be translated into the PQ node which no longer has the regulatory capacity of the reactive power. On the other hand, the node which voltage amplitude is out of limit will be placed on the ceiling, while the others' current value will be retained, and then converted into a PV bus.

Finally, the load nodes and the pure contact nodes will be directly translated into PQ nodes.

\section{Automatic adjustment of power flow}

The core essence of the power flow automatic adjust algorithm is that allocate the system active and reactive power shortfall to generator node in the system which still have the ability to regulate in accordance with the respective adjustment strategy. Based on the PQ decomposition, the automatic adjustment of the active and reactive power will be added to the iterative process, the specific process is shown in figure 2 .

\section{Example analysis}

In this paper, the IEEE 30 -bus test system ${ }^{[19]}$ is used to validate the algorithm mentioned in the text. In the process of power flow automatic adjusting algorithm, the voltage adjustable range is set to $[0.95,1.07]$.

In addition, in the example, set the dynamic changes of trend caused by the load increment of $5 \%$ to examine the adaptability of the algorithm.

According to the above conditions, the calculating results of the trend automatic adjustment algorithm are compared with the conventional power flow algorithm and the dynamic power flow algorithm ${ }^{[20]}$ calculations'. The comparative results are as follows(The added "*" nodes are Generator nodes).

Table 1. Comparison of bus voltage when load raises 5\%

\begin{tabular}{|c|c|c|c|}
\hline \multirow{2}{*}{ Nodes } & \multicolumn{3}{|c|}{ Bus voltage[pu] } \\
\cline { 2 - 4 } & $\begin{array}{c}\text { Conventional } \\
\text { algorithm }\end{array}$ & $\begin{array}{c}\text { Dynamic } \\
\text { algorithm }\end{array}$ & $\begin{array}{c}\text { Automatic adjustment } \\
\text { algorithm }\end{array}$ \\
\hline $1^{*}$ & 1.07000 & 1.07000 & 1.07000 \\
\hline $2^{*}$ & 1.07000 & 1.07000 & 1.05437 \\
\hline 3 & 1.06368 & 1.06410 & 1.06382 \\
\hline
\end{tabular}




\begin{tabular}{|c|c|c|c|}
\hline $5^{*}$ & 1.07000 & 1.07000 & 1.04953 \\
\hline $8^{*}$ & 1.07000 & 1.07000 & 1.05723 \\
\hline 10 & 1.06230 & 1.06256 & 1.04753 \\
\hline $11^{*}$ & 1.07000 & 1.07000 & 1.06120 \\
\hline $13^{*}$ & 1.07000 & 1.07000 & 1.05296 \\
\hline 18 & 1.03406 & 1.03433 & 1.02015 \\
\hline 26 & 1.03247 & 1.03263 & 1.01630 \\
\hline 27 & 1.06631 & 1.06643 & 1.03997 \\
\hline
\end{tabular}

Table 2.Comparison of generator nodes' active and reactive power when load raises $5 \%$

\begin{tabular}{|c|c|c|c|}
\hline \multirow{2}{*}{ Nodes } & \multicolumn{3}{|c|}{ Reactive power[pu] } \\
\cline { 2 - 4 } & $\begin{array}{c}\text { Conventional power } \\
\text { flow algorithm }\end{array}$ & $\begin{array}{c}\text { Dynamic power } \\
\text { flow algorithm }\end{array}$ & $\begin{array}{c}\text { Automatic adjustment } \\
\text { algorithm }\end{array}$ \\
\hline $1^{*}$ & -0.428988 & -0.400888 & -0.501253 \\
\hline $2^{*}$ & 0.182489 & 0.155375 & 0.139984 \\
\hline $5^{*}$ & 0.518999 & 0.508750 & 0.510743 \\
\hline $8^{*}$ & 0.561503 & 0.545474 & 0.423678 \\
\hline $11^{*}$ & 0.057029 & 0.056830 & 0.047234 \\
\hline $13^{*}$ & 0.136916 & 0.135298 & 0.141576 \\
\hline Total & 1.027950 & 1.000840 & 0.761962 \\
\hline
\end{tabular}

Table 3.Comparison of iteration and computing time when load raises 5\%

\begin{tabular}{|c|c|c|c|}
\hline & $\begin{array}{c}\text { Conventional power } \\
\text { flow algorithm }\end{array}$ & $\begin{array}{c}\text { Dynamic power } \\
\text { flow algorithm }\end{array}$ & $\begin{array}{c}\text { Automatic adjustment } \\
\text { algorithm }\end{array}$ \\
\hline Iteration times & 6 & 12 & 13 \\
\hline Computing time[s] & 0.015 & 0.016 & 0.073 \\
\hline
\end{tabular}

Table 1-3 show that the automatic adjustment used in this paper reduced the computational efficiency, but the computing time maintained an acceptable level; power adjustment reduced the total amount of reactive power generation of the system, achieved in situ reactive power balance, and improved the distribution of active and reactive power.

\section{Conclusion}

(1) Based on the PQ decomposition method, active and reactive power automatic adjusting method is introduced to the iterative process. According to the respective adjustment strategy, active and reactive power vacancies of the system are assigned to the generator nodes which still have the ability to regulate in the system to achieve automatic adjusting of the power flow;

(2) According to the specific association of the generator nodes and the surrounding load, the algorithm adjusts the node voltage and reactive power within a certain range to be more reasonable reactive power flow distribution to meet the balance requirements of the reactive area;

(3) This paper introduces the reactive power allocation factor. If the load changes, the system will adjust its reactive power according to the allocation factor to achieve the reactive-place balance, the system tends to be a more reasonable flow distribution.

\section{Acknowledgment}

Supported by National High Technology Research and Development Program 863(No: 2011AA05A119).

Supported by State Grid Corporation of China, Major Projects on Planning and Operation Control of Large Scale Grid(SGCC-MPLG029-2012). 


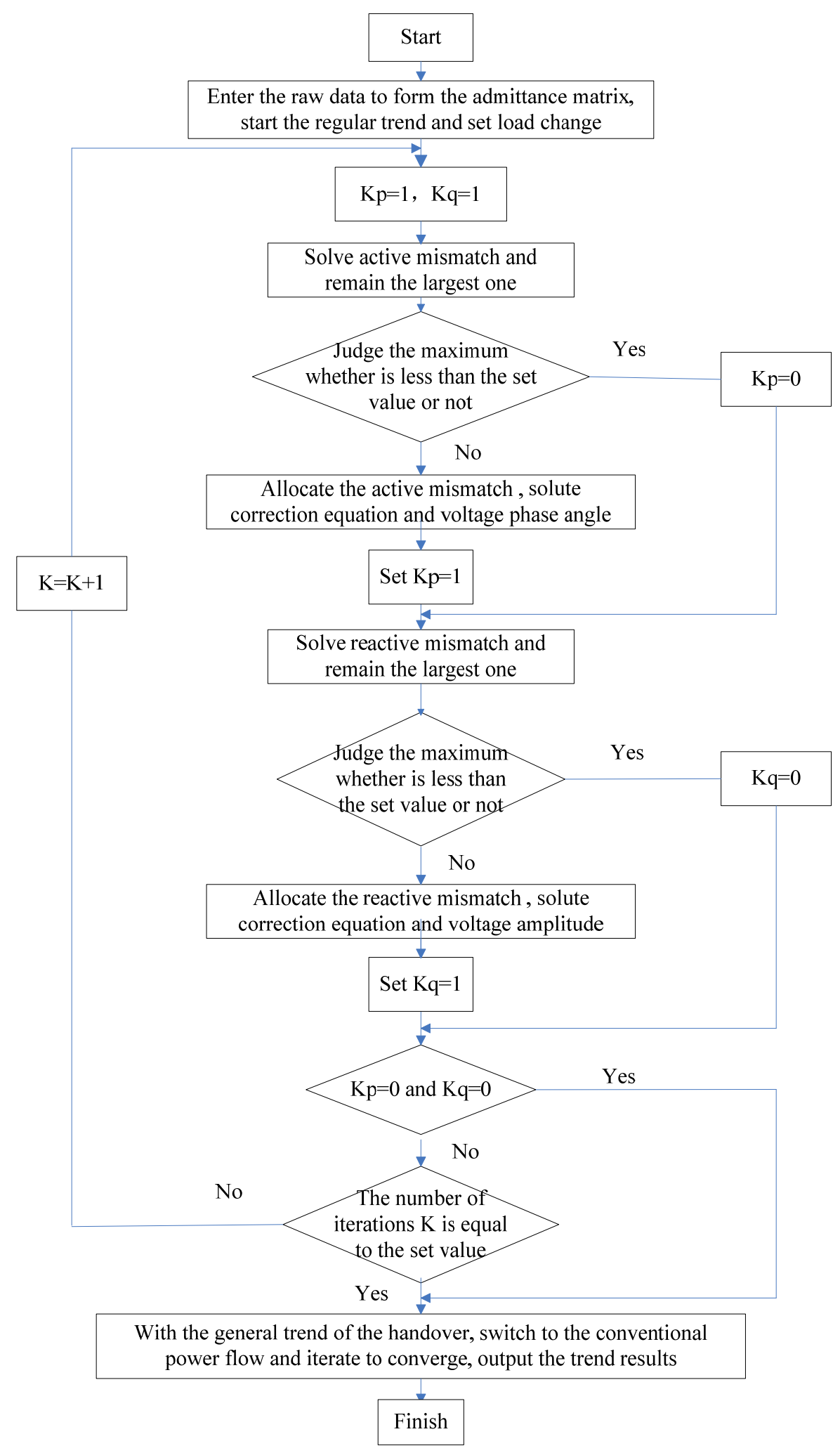

Fig. 2. The flow chart of the power flow automatic adjusting method

\section{References}

[1] Xi'an Jiaotong University, Tsinghua University,etc, Zhejiang University. Power system computation[M], Beijing : Water Conservancy and Electric Power Press, 1978.

[2] WU Ji-shun, HOU Zhi-jian. Power flow calculation method of the computer system [M]. Shanghai: Shanghai Jiaotong University Press, 2000.

[3] Le Nguyen H. Newton-Raphson method in complex form[J].IEEE Trans on Power Systems, 1997, 12(3):1355-1359. 
[4] Wang L,Li X R. Robust fast decoupled power flow[J]. IEEE Trans on Power Systems,2000, 15(1): 208-215.

[5] YUAN Gui-chuan,WANG Jian-quan,HAN Zhen-xiang. Optimal power flow under electricity market[J]. Power System Technology,2004,28(5):13-17.

[6] DING Xiao-ying, WANG Xi-fan, ZHANG Xian etc. Mixed integer optimal power flow based on interior point cutting plane method[J].Proceedings of the CSEE, 2004,24(2):1-7.

[7] WEI Hua, YANG Yu-de, LI Xiao-cong. Optimal power flow with multi-contingencies transient stability constrained[J]. Proceedings of the CSEE,2004,24(10):91-96.

[8] LIU Sheng-song, HOU Zhi-jian, JIANG Chuan-wen. Optimal power flow algorithm based on chaos optimization and linear interior point method[J].Power System Technology, 2003, 27(9): 23-28.

[9] Momoh J A, Zhu J Z.Improved interior point method OPF problems[J].IEEE Trans on Power Systems, 2000, 14(3): 1114-1120.

[10] ZHUO Jun-feng, ZHAO Dong-mei.A chaos optimization based algorithm for multi-objective fuzzy optimal power flow[J].Power System Technology, 2003,27(2): 41-44.

[11] Ruedu S M V,Almeida K C.Optimal power flow solutions under variable load conditions: reactive power cost modeling[A].IEEE Power Industry Computer Applications Conference [C]. Sydney,NSW: Institute of Electrical and Electronics Engineers lnc. 22-24 May 2001.300-305.

[12] Salamat SharifS, TaylorJ H,Hill E E,etal. Real-time implementation of optimal reactive power flow[J]. IEEE Power Engineering Review,2000,20(8);47-51

[13] ZHANG Hong-bin, WU Wen-chuan,ZHANG Bo-ming. Simulation study of the optimal control of the global reactive security constraints [J]. Automation of electric power systems, 1999, $23(5): 4-7,50$.

[14] WANG Yong-gang, HAN Xue-shan, LIU Chao. The dynamic optimal power flow [J]. Journal of Chinese electrical engineering, 1997, (3) : 195-198.

[15] QU Fu-di. Random flow calculation method based on the estimation of power system [D]. Northeast dianli university, 2011,1-3.

[16] Orero S U, Irvin g M N. A generic algorithm for network partition in Power system state estimation[A]. C KACC International Conference on Control 1996 [C]. Exeter ( UK ) : 1996, $162-165$.

[17] LIU Da-peng, TANG Guo-qing, CHEN Hang. Voltage to control the partition based on the Tabu search[J]. Automation of Electric Power Systems, 2002, 26 (6) : 18-22.

[18] LIU Yu-tian, Liang Jun, Du Zhengchun,etc. The neural network used for cutting machine control decision-making of the actual power system.Automation of Electric Power System and A Journal 1996, 8 (1) :19-23

[19] ZHANG Bo-ming,Chen Shou-sun.Higher power network analysis [M] .Beijing: Tsinghua University Press, 1996.

[20] YU Xiao-yan,YU Ji-lai.The dynamic trend based on the active and reactive power joint adjustment [J]. Power system technology, 2005, 29 (22) : 61-65 